\title{
Polarization-Independent Wideband High-Index-Contrast Grating Mirror
}

Bekele, Dagmawi Alemayehu; Park, Gyeong Cheol; Malureanu, Radu; Chung, II-Sug

Published in:

IEEE photonic Technology Letters

Link to article, DOI:

10.1109/LPT.2015.2438538

Publication date:

2015

Document Version

Peer reviewed version

Link back to DTU Orbit

Citation (APA):

Bekele, D. A., Park, G. C., Malureanu, R., \& Chung, I-S. (2015). Polarization-Independent Wideband High-IndexContrast Grating Mirror. IEEE photonic Technology Letters, 27(16), 1733-1736.

https://doi.org/10.1109/LPT.2015.2438538

\section{General rights}

Copyright and moral rights for the publications made accessible in the public portal are retained by the authors and/or other copyright owners and it is a condition of accessing publications that users recognise and abide by the legal requirements associated with these rights.

- Users may download and print one copy of any publication from the public portal for the purpose of private study or research.

- You may not further distribute the material or use it for any profit-making activity or commercial gain

- You may freely distribute the URL identifying the publication in the public portal 


\title{
Polarization-Independent Wideband High-Index-Contrast Grating Mirror
}

\author{
Dagmawi Alemayehu Bekele, Gyeong Cheol Park, Radu Malureanu, and Il-Sug Chung*
}

\begin{abstract}
Island-type two-dimensional high-index-contrast grating mirror based on a standard silicon-on-insulator wafer have been experimentally demonstrated. The measured spectra shows a bandwidth of $\sim 192 \mathrm{~nm}$ with a reflectivity over $99 \%$ as well as polarization independence. Numerical simulations show that the designed mirror has large tolerance to fabrication errors.
\end{abstract}

Index Terms-High index contrast, VCSEL, polarization, subwavelength grating.

\section{INTRODUCTION}

$\mathbf{T}$ HE grating structure referred to as high-index-contrast grating (HCG) is a single layer of near subwavelength grating with large refractive index difference from surrounding materials [1]. Since HCGs can exhibit high reflectivity over a broad wavelength range [2], [3], they have attracted much attention as an alternative to distributed Bragg reflectors (DBRs) especially for vertical-cavity surface-emitting laser (VCSEL) applications. By replacing one or both of the DBRs in a VCSEL with the HCG reflector, various interesting VCSEL structures with novel properties have been demonstrated [4][16]. The use of HCG reflector structure formed in the silicon (Si) layer of a silicon-on-insulator (SOI) wafer together with a direct wafer bonding with a III-V semiconductor with a gain material can innovate the fabrication processes of long wavelength VCSELs [8]-[11], [14]-[16], removing the need for growth of thick InP-lattice-matched DBRs or double wafer fusion of GaAs-lattice-matched DBRs [17]. Furthermore, it may bring novel characteristics including a high modulation speed over $100 \mathrm{~Gb} / \mathrm{s}$ [16] and a capability of emitting output light laterally into a Si waveguide [8], [11], [16] which is highly desirable for chip-level optical interconnects.

So far, most of studies on HCGs mentioned above have been focused on one-dimensional (1D) HCGs. These 1D HCG reflectors are polarization-dependent due to the asymmetry of the grating profile for transverse electric (TE) and transverse magnetic (TM) polarizations and hence can provide a high reflectivity to a specific polarization at a given wavelength. This polarization selectivity is an advantage for VCSELs for communication applications that have been of most interest so far. However, recently, there is increasing need for polarization-independent HCGs, which requires twodimensional (2D) HCG. For example, Tsunami et al. have

The authors are with the Department of Photonics Engineering (DTU Fotonik), Technical University of Denmark, DK-2800 Kgs. Lyngby, Denmark. (*e-mail: ilch@fotonik.dtu.dk)

The authors gratefully acknowledge support from the Danish Council for the Independent Research through FTP program (Grant No. 0602-01885B).

Manuscript received February 19, 2015; revised March 17, 2015.

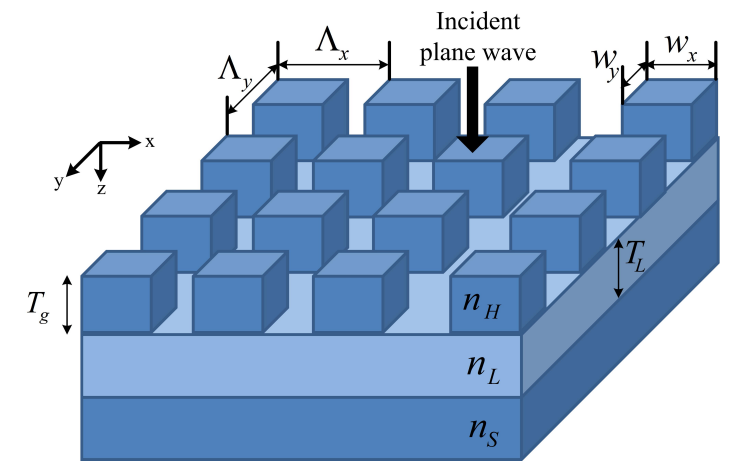

Fig. 1. Schematic diagram of an island-type 2D HCG. $T_{g}$ : grating thickness, $n_{H}$ : grating refractive index, $\Lambda_{x, y}$ : period in $x$ or $y$ direction, $w_{x, y}$ : grating width in $x$ or $y$ direction, $n_{L}$ : refractive index of a layer underneath the grating, $T_{L}$ : thickness of the layer underneath the grating, $n_{S}$ : refractive index of substrate.

proposed to use 2D HCG reflectors on a SOI wafer to realize a 2D network of VCSEL-based optical buffer memories [14]. In this proposed structure, the polarization independence of the 2D HCG reflectors is essential to achieve polarization-bistable oscillations and swtiching in the 2D-HCG based VCSELs [18]. In VCSEL-based Doppler velocimetry applications [19], 2D HCGs has a potential to innovate the device structure.

Recently, several types of 2D HCGs have been studied, aiming at polarization-independent wideband reflectors with reflectivity $R \geq 99 \%$. They include a crossed type [14], [20], an island type [21], and a two-layered cross-stacked type [22]. For the two-layered type reflector consisting of two layers of 1D HCGs, a broad bandwidth of $200 \mathrm{~nm}$ has been demonstrated. However, its complex fabrication steps could be a drawback and it appears mechanically unstable for a direct wafer bonding. For the crossed type 2D HCG where two 1D HCGs are connected to each other in a single layer, a broad bandwidth of $200 \mathrm{~nm}$ and an integration with a VCSEL have been demonstrated, showing that this type is promising. For the island type (c.f., Fig. 1), a numerical study has shown that an even broader bandwidth of $220 \mathrm{~nm}$ is achievable and there has been no experimental study yet. Furthermore, there has been no rigorous investigation for either the crossed type or the island type, on the effect of fabrication errors on the 2D HCG properties by considering all probable fabrication errors simultaneously.

These have motivated the experimental investigation of the polarization-independent properties of island type 2D HCGs as well as the numerical investigation of the influence of fabrication errors presented in this paper. 


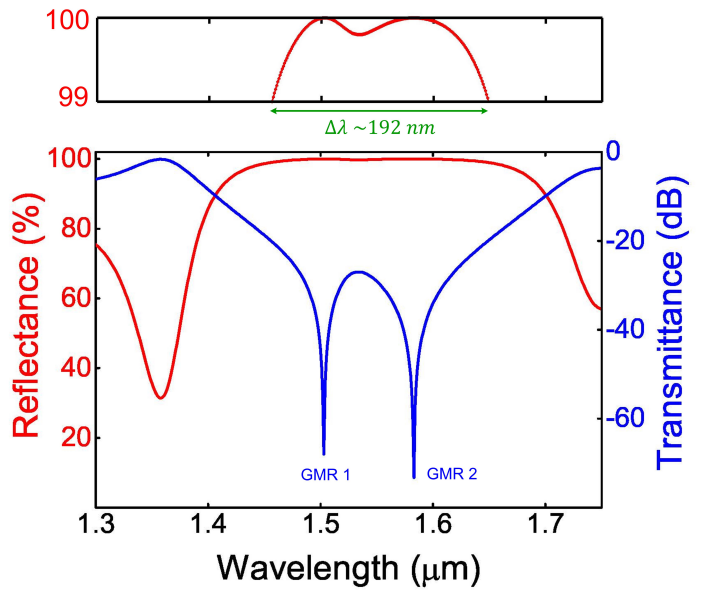

Fig. 2. Calculated reflectance and transmittance spectrum of an optimal structure $\Lambda_{x}=700 \mathrm{~nm}, w_{x}=410 \mathrm{~nm}$.

\section{DESIGN AND FABRICATION TOLERANCE STUDY}

Fig. 1 shows a schematic diagram of an island type 2D HCG with design parameters such as grating refractive index $\left(n_{H}\right)$, refractive index of the layer underneath the grating $\left(n_{L}\right)$, refractive index of the substrate $\left(n_{S}\right)$, grating layer thickness $\left(T_{g}\right)$, thickness of the layer below the grating $\left(T_{L}\right)$, grating period in $x$ or $y$ direction $\left(\Lambda_{x, y}\right)$, and grating width in $x$ or $y$ directions $\left(w_{x, y}\right)$. Some of the design parameters are determined from the standard SOI wafer used for fabrication. These include: $n_{H}=3.47, n_{L}=1.47, n_{S}=3.47, T_{g}=500 \mathrm{~nm}$, and $T_{L}=3$ $\mu \mathrm{m}$. Furthermore, to guarantee polarization-independence, the grating should be symmetric after $90^{\circ}$ rotation. This requirement demands that $\Lambda_{x}=\Lambda_{y}$ and $w_{x}=w_{y}$. Additionally, it is assumed that the region above the grating is air, all the refractive indices are isotropic and non-dispersive, and the incidence angle $\left(\theta_{\text {inc }}\right)$ is zero.

Firstly, with these pre-conditions, 2D numerical simulations based on rigorous coupled-wave analysis (RCWA) [23] are performed to find optimum parameter sets, i.e., $\left(w_{x}, \Lambda_{x}\right)$. In order to ensure the accuracy of calculation, 60 diffraction orders were considered. Fig. 2 shows the calculated reflectance and transmittance spectra for an optimum parameter set. As typically observed in 1D HCGs, there are two dips in the transmission spectrum within the stop band, which correspond to two guided mode resonances (GMRs). The bandwidth with reflectivity higher than $99 \%$ is $\sim 192 \mathrm{~nm}$ for the wavelength range of $1458 \mathrm{~nm}$ to $1650 \mathrm{~nm}$.

Then, the tolerance of reflection properties over fabrication errors are estimated for the optimal set. Geometrical parameters, i.e., the grating period, grating thickness, and grating width are separately varied around the optimal grating period of $700 \mathrm{~nm}$, the Si layer thickness of a standard SOI wafer of $500 \mathrm{~nm}$, and the optimal grating width of $410 \mathrm{~nm}$ respectively. While one parameter is varied, the others are kept constant. The results are shown in Fig. 3. Allowed parameter variations while still achieving reflectivity, $R \geq 99 \%$ and bandwidth, $\Delta \lambda \sim 192 \mathrm{~nm}$, are evaluated by changing the parameter values centered around the optimum parameter values. It is found that both grating thickness and width have \pm 20

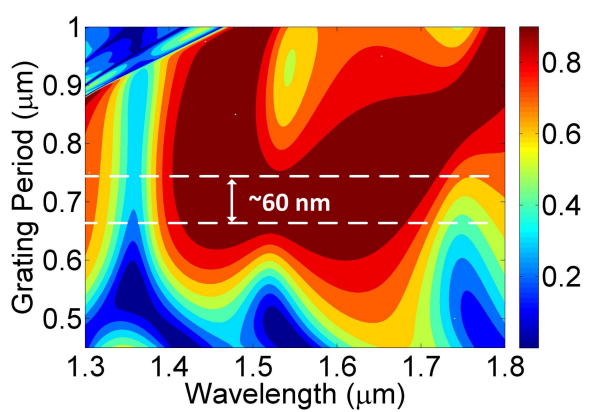

(a)

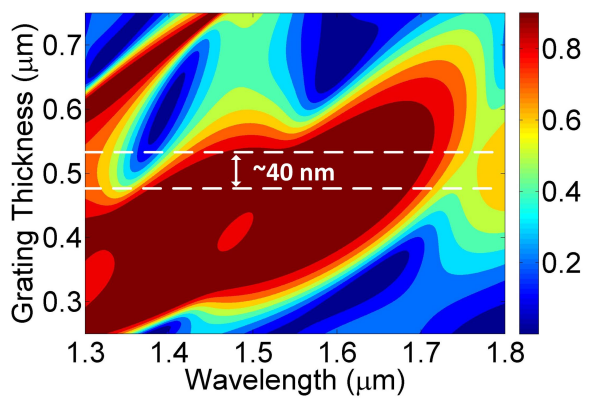

(b)

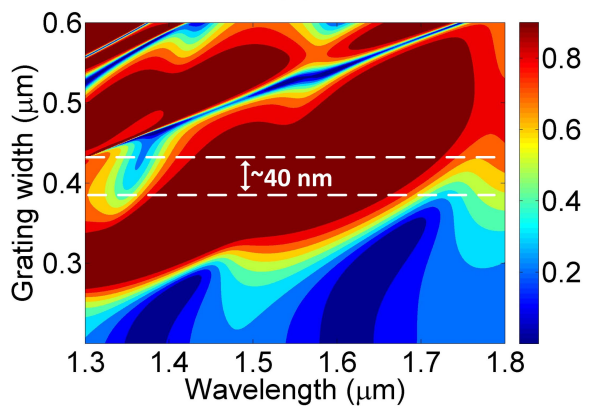

(c)

Fig. 3. Reflectivity as a function of (a) grating period, (b) grating thickness, and (c) grating width as well as wavelength.

$\mathrm{nm}$ tolerance while the grating period has $\pm 30 \mathrm{~nm}$. These tolerances are achievable within the accuracy limit of e-beam lithography and dry etching processes. We need to note that the tolerance analysis presented in Fig. 3 has simplified the real situation by assuming all other parameters except for one are constant. However, in reality all the grating parameters can vary simultaneously, uncorrelated to each other during the fabrication processes. To further investigate these effects a more rigorous analysis based on Monte Carlo simulation [24] is implemented.

Monte Carlo simulation is performed to explore sensitivity of design parameters for 1000 sample parameter sets each with random $\Lambda_{x}, \Lambda_{y}, w_{x}$ and $w_{y}$ values having Gaussian distribution, as shown in the histogram plots in Figs. 4(a) to 4(d). All the distributions are centered around the optimum parameter values with a standard deviation $(\sigma)$ of $5 \mathrm{~nm}$, which is a probable value occurring in fabrication processes. The simulation results presented in Figs. 4(e) and 4(f) show that most of samples have a reflectivity higher than $99.5 \%$ at $1.55 \mu \mathrm{m}$ and a bandwidth wider than $180 \mathrm{~nm}$. This indicates 

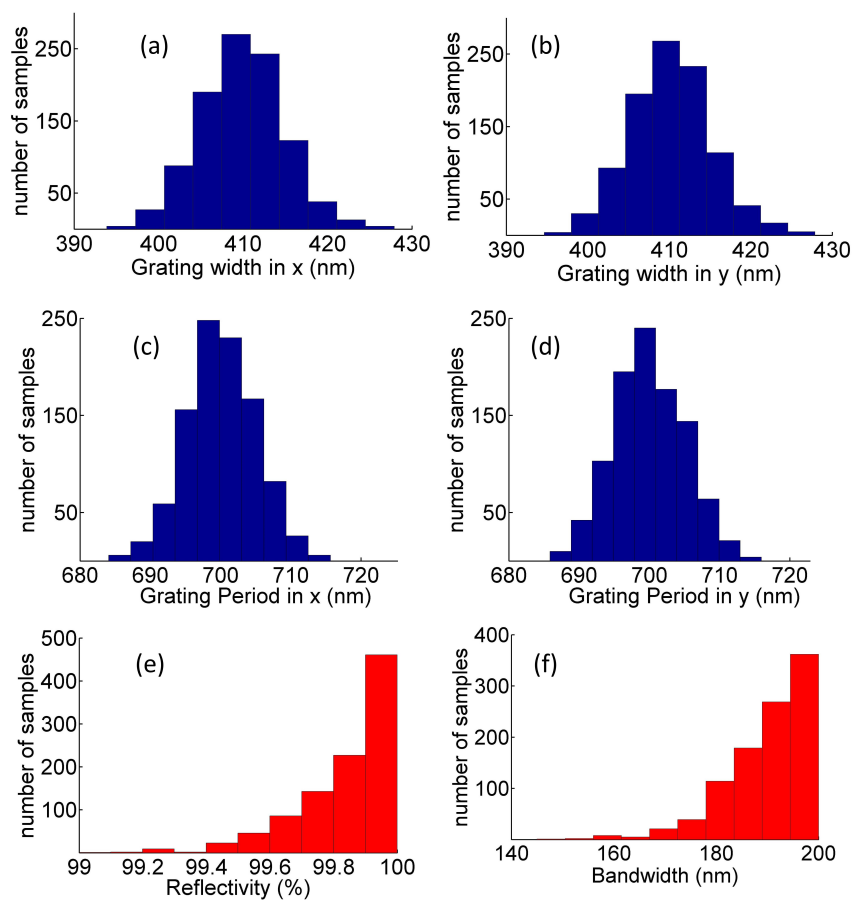

Fig. 4. Fabrication sensitivity analysis with Monte Carlo method. Histrogram plot of (a) Grating width in $\mathrm{x}$ direction (b) Grating width in y direction (c) Grating period in $\mathrm{x}$ direction (d) Grating period in $\mathrm{y}$ direction. Monte Carlo analysis results for (e) reflectivity and (f) bandwidth of 2D HCG.

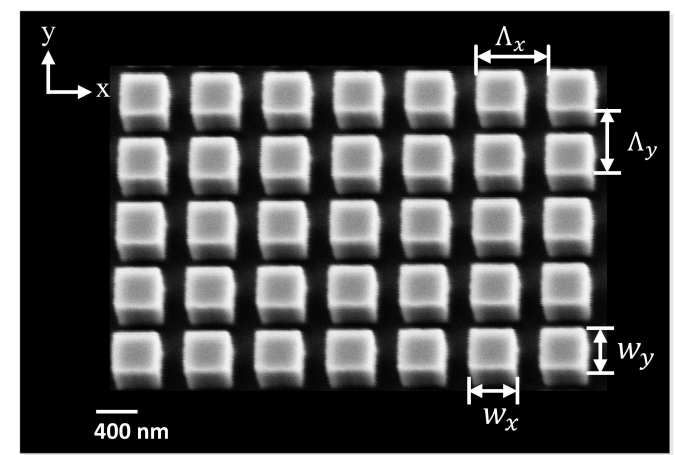

Fig. 5. Scanning electron microscope (SEM) image of the fabricated $2 \mathrm{D}$ HCG mirror. Grating is formed in the Si layer of a SOI wafer.

that the island-type 2D HCG design found in this study is robust to uncorrelated variation of parameters introduced during fabrication processes.

\section{RESUlTS AND Discussion}

Samples were prepared by electron beam lithography (JEOL JBX-9500FS) and dry etching processes. The dry etching of silicon involves etching and passivation phases in a cyclic process called Bosch process. A scanning electron microscope image of the fabricated sample tilted at an angle is shown in Fig. 5. The side walls of square islands show vertical profile indicating successful etching of the crystalline silicon. Additionally, the samples show smooth surfaces with etching roughness less than few nanometers.

The characterization of the device was done by illuminating super-continuum light with a beam diameter of approximately

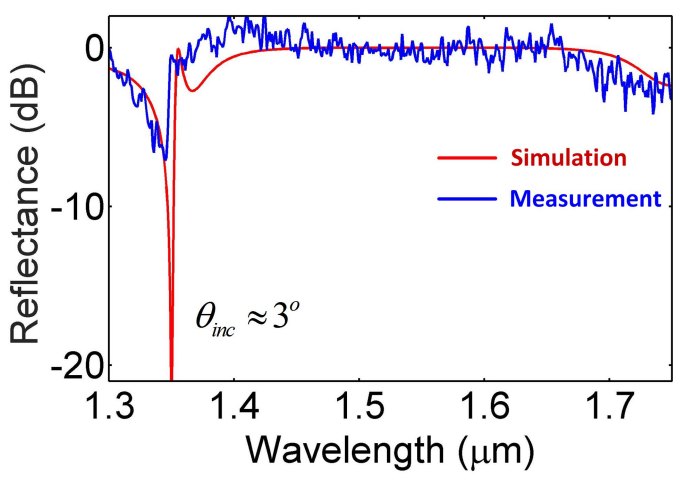

(a)

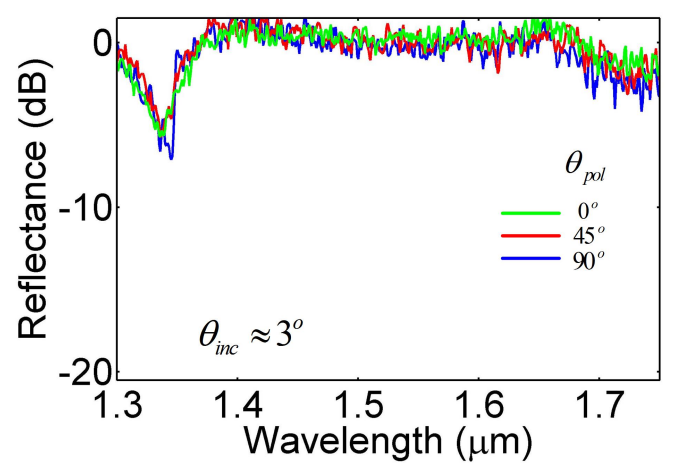

(b)

Fig. 6. Reflectance spectrum of incident plane wave at $\theta_{\text {inc }} \sim 3^{\circ}$. (a) Simulation and measurement reflectance spectra for TM polarization. (b) Measurement reflectance spectra at different linear polarization angles $\left(\theta_{\mathrm{pol}}\right)$.

$300 \mu \mathrm{m}$ with a spectral range of $1300 \mathrm{~nm}$ to $1750 \mathrm{~nm}$, onto the grating area of $500 \mu \mathrm{m} \times 500 \mu \mathrm{m}$ at an incident angle of $\theta_{\text {inc }} \approx 3^{\circ}$. This angle of incidence is introduced to separate the optical paths of the incoming and reflected waves. The reflected light from the grating was collected by a parabolic mirror and coupled to a multimode fiber which fed the light to an optical spectrum analyzer (Ando AQ6317B). The spectrum was then normalized with respect to the reflectance spectrum of a reference aluminum mirror.

In Fig. 6(a), the measured and calculated reflectance spectra for $\mathrm{TM}$ polarization are plotted at $\theta_{\mathrm{inc}} \approx 3^{\circ}$ in $\mathrm{dB}$ scale, showing a good agreement. Calculated reflectance spectra for all linear polarization angles are the same as the simulated spectrum shown at TM polarization. It is noteworthy that there is a sharp resonance peak around $1355 \mathrm{~nm}$, which is not found in the spectrum of Fig. 2 calculated for $\theta_{\text {inc }}=0^{\circ}$. When the incident plane wave impinges at an oblique incidence, odd symmetry modes that cannot be excited at normal incidence due to symmetry, can be excited [25]. This sharp resonance peak corresponds to the excitation of odd modes with a relatively high $Q$ factor. Small difference in peak wavelengths between the measured and calculated spectrum can be attributed to the neglected dispersion in calculations.

Fig. 6(b) shows the measured reflecance spectra at three linear polarization angles $\left(\theta_{\mathrm{pol}}\right)$. The TM and TE polarizations 
correspond to $\theta_{\mathrm{pol}}=0^{\circ}$ and $90^{\circ}$, respectively. The overall reflection spectrum and resonance peak are maintained for all linear polarization angles. Note that the measurement at $\theta_{p o l}=90^{\circ}$ is in turn compared with the calculated reflectance spectrum in Fig. 6(a). Therefore, we may conclude that the fabricated sample shows polarization-independent reflectivity.

\section{CONCLUSION}

We have numerically designed and experimentally demonstrated an island-type 2D HCG on a standard SOI wafer. The fabricated sample exhibits a broadband polarizationindependent reflection spectra. Numerical investigations show that this island-type 2D grating structure has good tolerance over fabrication errors. This island-type 2D HCG reflector appears promising for long wavelength VCSELs integrated onto a SOI wafer.

\section{REFERENCES}

[1] C. Mateus, M. Huang, L. Chen, C. Chang-Hasnain, and Y. Suzuki, "Broad-band mirror (1.12-1.62 $\mu \mathrm{m})$ using a subwavelength grating," IEEE Photon. Technol. Lett., vol. 16, no. 7, pp. 1676-1678, July 2004.

[2] R. Magnusson and M. Shokook-Saremi, "Physical basis for wideband resonant reflectors," Opt. Express, vol. 16, no. 5, pp. 3456-3462, 2008.

[3] V. Karagodsky, F. G. Sedgwick, and C. J. Chang-Hasnain, "Theoretical analysis of sub wavelength high contrast grating reflectors," Opt. Express, vol. 18, no. 16, pp. 16973-16988, 2010.

[4] M. C. Y. Huang, Y. Zhou, and C. J. Chang-Hasnain, "A surface-emitting laser incorporating a high-index-contrast subwavelength grating," Nat. Photonics, vol. 1, no. 2, pp. 119-122, 2007.

[5] S. Boutami, B. Benbakir, J.-L. Leclercq, and P. Viktorovitch, "Compact and polarization controlled $1.55 \mu \mathrm{m}$ vertical-cavity surface-emitting laser using single-layer photonic crystal mirror," Appl. Phys. Lett., vol. 91, p. 071105, 2007.

[6] Y. Z. Michael C. Y. Huang and C. J. Chang-Hasnain, "A nanoelectromechanical tunable laser," Nat. Photonics, vol. 2, p. 180, Mar. 2008.

[7] I.-S. Chung, V. Iakovlev, A. Sirbu, A. Mereuta, A. Caliman, E. Kapon, and J. Mørk, "Broadband MEMS-tunable high-index-contrast subwavelength grating long-wavelength VCSEL," IEEE J. Quantum Electron., vol. 46, no. 9, pp. 1245-1253, Sept. 2010.

[8] I.-S. Chung and J. Mørk, "Silicon-photonics light source realized by IIIV/Si grating-mirror laser," Appl. Phys. Lett., vol. 97, no. 15, p. 151113 , 2010.

[9] C. Sciancalepore, B. B. Bakir, X. Letartre, J.-M. Fedeli, N. Olivier, D. Bordel, C. Seassal, P. Rojo-Romeo, P. Regreny, and P. Viktorovitch, "Quasi-3D light confinement in double photonic crystal reflectors VCSELs for CMOS-compatible integration," J. Lightwave Technol., vol. 29, no. 13 , pp. 2015-2024, 2011

[10] C. Sciancalepore, B. B. Bakir, X. Letartre, J. Harduin, N. Olivier, C. Seassal, J.-M. Fedeli, and P. Viktorovitch, "CMOS-compatible ultracompact $1.55-\mu \mathrm{m}$ emitting vcsels using double photonic crystal mirrors," IEEE Photon. Technol. Lett., vol. 24, no. 6, pp. 455-457, 2012.

[11] P. Viktorovitch, C. Sciancalepore, B. B. Bakir, X. Letartre, and C. Seassal, "Double photonic crystal vertical-cavity surface-emitting lasers," Proc. SPIE, vol. 8633, p. 863302, 2013.

[12] Y. Rao, W. Yang, C. Chase, M. C. Y. Huang, D. P. Worland, S. Khaleghi, M. R. Chitgarha, M. Ziyadi, A. E. Willner, and C. J. Chang-Hasnain, "Long-wavelength VCSEL using high-contrast grating," IEEE J. Sel. Top. Quantum Electron., vol. 19, no. 4, p. 1701311, 2013.

[13] S. Inoue, J. Kashino, A. Matsutani, H. Ohtsuki, T. Miyashita, and F. Koyama, "Highly angular dependent high-contrast grating mirror and its application for transverse-mode control of VCSELs," Jpn. J. Appl. Phys., vol. 53, p. 090306, 2014.

[14] Y. Tsunemi, N. Yokota, S. Majima, K. Ikeda, T. Katayama, and H. Kawaguchi, "1.55 $\mu \mathrm{m}$ VCSEL with polarization-independent HCG mirror on SOI," Opt. Express, vol. 21, no. 23, pp. 28685-28 692, Nov. 2013.

[15] J. Ferrara, W. Yang, L. Zhu, P. Qiao, and C. J. Chang-Hasnain, "Heterogeneously integrated long-wavelength VCSEL using silicon high contrast grating on an SOI substrate," Opt. Express, vol. 23, no. 3, p. $2512,2015$.
[16] G. C. Park, W. Xue, A. Taghizadeh, E. Semenova, K. Yvind, J. Mørk, and I.-S. Chung, "Hybrid vertical-cavity laser with lateral emission into a silicon waveguide," Laser Photonics Rev., doi: 10.1002/lpor.201400418.

[17] E. Kapon and A. Sirbu, "Long-wavelength VCSELs: Power-efficient answer," Nat. Photon., vol. 3, pp. 27-29, 2009.

[18] H. Kawaguchi, T. Mori, Y. Sato, and Y. Yamayoshi, "Optical buffer memory using polarization-bistable vertical-cavity surface-emitting lasers," Jpn. J. Appl. Phys., vol. 45, no. 9L, p. L894, 2006.

[19] J. Albert, M. Soriano, I. Veretennicoff, K. Panajotov, J. Danckaert, P. Porta, D. Curtin, and J. McInerney, "Laser doppler velocimetry with polarization-bistable VCSELs," IEEE J. Select. Topics Quantum Electron., vol. 10, no. 5, pp. 1006-1012, Sept 2004.

[20] K. Ikeda, K. Takeuchi, K. Takayose, I.-S. Chung, J. Mørk, and H. Kawaguchi, "Polarization-independent high-index contrast grating and its fabrication tolerances," Appl. Opt., vol. 52, no. 5, pp. 10491053, Feb. 2013.

[21] R. G. Mote, S. F. Yu, W. Zhou, and X. F. Li, "Design and analysis of two-dimensional high-index-contrast grating surface-emitting lasers," Opt. Express, vol. 17, no. 1, pp. 260-265, Jan. 2009.

[22] D. Zhao, H. Yang, Z. Ma, and W. Zhou, "Polarization independent broadband reflectors based on cross-stacked gratings," Opt. Express, vol. 19, no. 10, pp. 9050-9055, May 2011.

[23] L. Li, "Formulation and comparison of two recursive matrix algorithms for modeling layered diffraction gratings," J. Opt. Soc. Am. A, vol. 13, no. 5, pp. 1024-1035, May 1996.

[24] A. Taghizadeh, G. C. Park, J. Mørk, and I.-S. Chung, "Hybrid grating reflector with high reflectivity and broad bandwidth," Opt. Express, vol. 22, no. 18, pp. $21175-21184$, Sep. 2014

[25] W. Yang and C. J. Chang-Hasnain, "Physics of high contrast gratings: a band diagram insight," Proc. SPIE, vol. 8633, pp. 863303-1, 2013. 